\title{
Intestinal goblet cell carcinoid presenting with recurrent sterile peritonitis in a patient on peritoneal dialysis: a case report
}

\author{
Chih-Wei Chen", Jan-Show Chu², Li-Chun Hsieh ${ }^{3,4}$, Chih-Chin Kao ${ }^{1,5}$, Yen-Chung Lin ${ }^{1,5}$ and Hsi-Hsien Chen ${ }^{1, *^{*}}$
}

\begin{abstract}
Background: Goblet cell carcinoid is a rare variant of appendiceal carcinoid with mixed endocrine and exocrine features. The most common symptom and signs are abdominal pain, acute appendicitis and palpable mass. Additionally, abdominal pain is common in patient on peritoneal dialysis, which may confound the diagnosis in such patient.

Case presentation: We report a 71- years- old woman on peritoneal dialysis that experienced several episodes of abdominal cramping pain and sterile peritonitis. She had one episode of severe pain and underwent an appendectomy for suspicion of appendicitis. Goblet cell carcinoid was diagnosed. She had no further abdominal pain after she received appendectomy.

Conclusions: Malignant dialysate was rarely reported in patient with peritoneal dialysis. However, goblet cell carcinoid can initially present with acute appendicitis, chronic intermittent abdominal pain and mimicking peritonitis. In systemically reviewing the literature, this is the first case report of sterile peritonitis with peritoneal dialysis caused by goblet cell carcinoid.
\end{abstract}

Keywords: Case report, Goblet cell carcinoid, Sterile peritonitis, Peritoneal dialysis

\section{Background}

Goblet cell carcinoid is a rare variant of appendiceal carcinoid with mixed endocrine and exocrine features. The most common clinical presentation is acute appendicitis $[1,2]$. Typically, GCC tumors are infiltrative and involve the entire appendix circumferentially [3]. Nevertheless, Tang et al. [1] reported that abdominal pain and palpable mass are also reported presented in $50 \%$ of GCC patients. Additionally, abdominal pain is common in patients on peritoneal dialysis, which may confound the diagnosis in such patient. For these patients, right hemicolectomy has been traditionally suggested by physician [2]. We performed systematic review of PubMed and Cochrane Library databases and noted no similar cases of goblet cell carcinoid causing abdominal pain which mimics peritoneal dialysis related peritonitis.

\footnotetext{
* Correspondence: 570713@yahoo.com.tw

'Division of Nephrology, Department of Internal Medicine, Taipei Medical University Hospital, Taipei, Taiwan

${ }^{5}$ Division of Nephrology, Department of Internal Medicine, School of Medicine, College of Medicine, Taipei Medical University, Taipei, Taiwan Full list of author information is available at the end of the article

We report this unique case of GCC presenting with sterile peritonitis in a patient on peritoneal dialysis to prompt clinicians to consider GCC in the differential diagnosis of similar symptoms in such patients.

\section{Case presentation}

A 71- years- old woman with underlying hypertension and end- stage renal disease on peritoneal dialysis, experienced several episodes of abdominal cramping pain with mild diarrhea 3 months after starting peritoneal dialysis. She had chills but no fever. A physical examination showed lower abdominal tenderness, but no rebounding pain. Initial complete blood count showed no leukocytosis $(8400 / \mu \mathrm{L})$. However, the initial dialysate studies showed increasing leukocytes with a predominance of neutrophils in first few sample, shifting to lymphocyte predominance in later sample (Table 1). Dialysate and blood cultures from 15 sets over 4 months since initial abdominal pain showed no growth. Since empirical antibiotic treatment did not improve the patient's condition of recurrent cramping pain, abdominal computerized tomography (CT) was performed which 
Table 1 Serial dialysate analysis of patient during episodes of abdominal pain in 2012

\begin{tabular}{lllllllll}
\hline Date & $09 / 13$ & $09 / 16$ & $09 / 19$ & $09 / 21$ & $09 / 25$ & $10 / 02$ & $10 / 12$ & $11 / 19$ \\
\hline Specific gravity & 1.010 & 1.018 & 1.010 & 1.010 & 1.014 & 1.014 & 1.010 & 1.010 \\
Red blood cells $/ \mathrm{mm}^{3}$ & 6 & 180 & 63 & 297 & 126 & 81 & 75 & 9 \\
White blood cells $/ \mathrm{mm}^{3}$ & 136 & 648 & 243 & 90 & 144 & 222 & 252 & 494 \\
Neutrophil:Lymphocyte:H:M & $24: 21: 48: 2: 5(\mathrm{E})$ & $80: 20$ & $48: 16: 28: 8$ & $24: 27: 48: 1$ & $35: 32: 22: 4: 7$ & $10: 81: 1: 8(\mathrm{E})$ & $4: 92: 4$ & $44: 53: 1: 2$ \\
\hline
\end{tabular}

$H$ macrophage, $M$ mesothelial, $E$ Eosinophil

revealed no abnormality. The patient still complained intermittent abdominal pain after the $\mathrm{CT}$, so pain control medication (including acetaminophen and tramadol) was prescribed for the patient. The Tenckhoff catheter was removed 2 months after the onset of abdominal pain, and then hemodialysis was started. Laparotomy and biopsy of peritoneal were performed during removal of Tenckoff catheter. Staining for acid-fast bacilli was negative. She continued to complain of intermittent abdominal cramping pain. One and half year after her initial abdominal pain, abdominal CT was again performed due to an episode of particularly severe abdominal pain. The scan showed fluid collection in the right pelvic cavity extending to the distal part of the appendix (Fig. 1). An appendectomy was immediately performed, and goblet cell carcinoid (GCC) with suppurative appendicitis was diagnosed. The CT scan before patient received appendectomy show the swollen appendix without obvious extraappendiceal lesion. On the other hand, according to the surgical and histopathological findings, the appendix was perforated with adhesion to

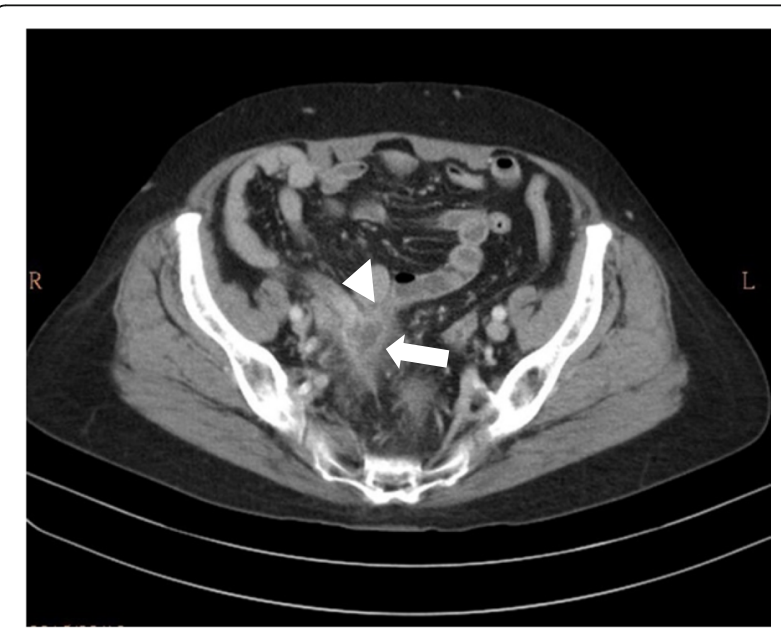

Fig. 1 Contrast-enhanced pelvic computed tomography. The scan reveals one $2.1 \times 1.6 \mathrm{~cm}$ irregular-shaped cystic mass (arrow) with marginal enhancement in the right pelvic cavity, abutting the swollen appendix (arrowhead). The tumor is located at the tip of appendix with possible subserosa invasion and attaches to the right pelvic side wall. The scan also shows inflammatory change with regional ileal bowel loop adhesion to the mass. Some fluid collection around the tumorbowel complex is noted ileum, accompanied by regional inflammatory changes in pelvic cavity. Microscopically, the infiltrating neoplastic cells were found in the appendiceal wall, including mucosa, submucosal, muscular propria, and subserosal layers. The infiltrated wall also showed fibrotic change. No evidence extra-appendiceal invasion of the tumor could be found (Fig. 2, the pathology specimen of this patient). Right hemicolectomy and adjuvant chemotherapy were suggested for complete treatment of GCC but the suggestions were refused by the patient. No further abdominal pain has been noted since appendectomy, and the patient still visits oncologist outpatient department for regular follow up without evident of recurrent tumor.

\section{Discussion and Conclusions}

Peritonitis in during peritoneal dialysis is a significant complication in clinically which can cause death or structural changes of the peritoneum [4]. Peritoneal infection is also a common cause of dialysis patients switching the therapy from peritoneal dialysis to hemodialysis [5]. On the other hand, sterile peritonitis is a common issue in the patients receiving peritoneal dialysis. Sterile peritonitis, culture-negative peritonitis, is often caused by antecedent empirical antibiotic treatment before dialysate culture are collected for culture. Additionally, infection caused by atypical organisms or non-infectious causes, can also lead to sterile peritonitis [6]. Peritoneal dialysate cultures prove to be negative in up to $22 \%$ of case [6]. Patients using icodextrin for peritoneal dialysis often develop peritonitis with an associated non-neutrophil predominant increasing in dialysate white blood cells [7]. Tuberculous (TB) peritonitis and malignancy related peritonitis also cause similar changes [6]. However, non-neutrophil-dominant increases in dialysate white blood cells in association with malignancy dialysate are rare $[8,9]$. Lymphoma sometimes mimics peritonitis in patients on ambulatory peritoneal dialysis [8]. Peritoneal metastases can also mimic peritonitis [9].

Goblet cell carcinoid (GCC), an unique and enigmatic tumor involving the appendix almost exclusively, is a rare variant of appendiceal carcinoid [10]. Carcinoid tumors account for up to $85 \%$ of all appendiceal tumors, white, GCC accounts for only $6 \%$ of all appendiceal 

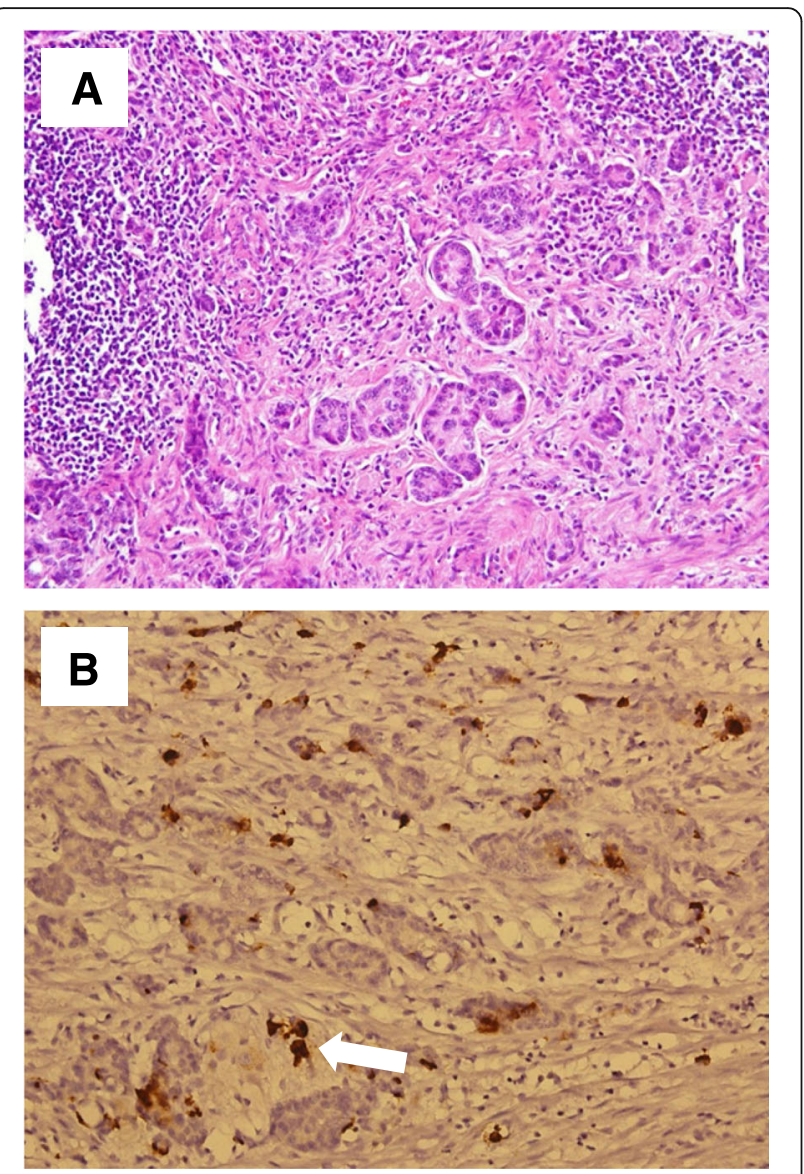

Fig. 2 Peritoneum obtained by laparotomy. (200X) a H \& E staining shows typical goblet cell carcinoid. The photomicrography shows goblet cell carcinoid composed of concentric infiltrating neoplastic cells in the appendiceal wall, including the mucosa, submucosa, muscular propria, and subserosal layers. The infiltrated wall shows fibrotic change. The tumor includes small solid or tubule-like tumor clusters, displaying monotonous nuclei with fine chromatin and eosinophilic cytoplasm, and occasional mucin-producing goblet cells arranged singly or small nests. $\mathbf{b}$ The Immunohistochemical study is positive for chromogranin A Brown color (arrow)

carcinoid tumors [11]. The term GCC was firstly introduced by Subbuswamy et al. in 1974 [12]. GCC derive from a pluripotent intestinal stem cell that differentiates into both mucinous and neuroendocrine cells [13]. Thus, it shares histologic features of both adenocarcinomas and carcinoid tumors. Additionally, GCC is reportedly more aggressive and unpredictable in nature than other carcinoid tumors.

The most common clinical presentation is acute appendicitis [1]. Abdominal pain and a palpable mass are also reported by $50 \%$ of GCC patients [2]. Pham et al. [1]. also reported other symptoms including bowel obstruction, intussusception, gastrointestinal bleeding, and chronic intermittent lower abdominal pain. Compared with other carcinoid tumors which are usually asymptomatic, GCC often presents with clinical symptoms. Thus, the incidental diagnostic rate is only about 3\% [2].
Despite serotonin can be detected immunohistochemically in GCC tumor [14], there is no carcinoid syndrome has been reported in GCC patients and urinary 5hydroxyindoleacetic acid (5-HIAA) levels in these patients are usually within normal limits, as opposed to carcinoid tumors, which are commonly with elevated urinary 5-HIAA and serum chromogranin A [15].

The most common route of metastases is transcoelomic/peritoneal invasion, and the most common sites involved are ovaries, and the peritoneal surfaces of the pelvis and abdominal cavity [10].

The natural history of patients with GCC is intermediate in aggressiveness between adenocarcinomas and classical carcinoids [10]. Based on its natural history and malignant nature, optimal therapeutic startegies are in general similar to adenocarcinomas rather than classical carcinoids [10]. Thus, surgical management with right hemicolectomy is recommended after appendectomy for most cases, particularly those with an adenocarcinoma components [2]. Nevertheless, right hemicolectomy is a significant abdominal procedure with an associated risk, especially in the infirm or elderly [15]. Peritoneal carcinomatosis is the most common cause of death [10]. Goblet cell carcinooids are associated with a 60\% 10-year survival and is similar to survival after treatment of a low-risk adenocarcinoma [15]. The prognosis of GCC is intermediate between appendiceal carcinoids and adenocarcinomas [10].

Our patient, who experienced abdominal pain intermittently for months had been diagnosed with peritonitis related to peritoneal dialysis. Given a clinical history of recurrent sterile peritonitis with a predominance of lymphocytes in the dialysate, an atypical infection (including tuberculosis) and intra-abdominal malignancy must be considered in the differential diagnosis. However, no dialysate or ascites was sent to cytology analysis in our patient. We also considered intermittent bowel obstruction as the cause of abdominal pain, which was the common presentation of GCC [1], but there were no sufficient evidence to support the possibility.

Of all the carcinoid tumors, GCC, in particular, can initially present with acute appendicitis, chronic intermittent abdominal pain, and malignant ascites mimicking peritonitis related to peritoneal dialysis in such patient. Through systematic review of PubMed and Cochrane Library databases, there were no similar cases reported. We report this unique case of GCC presenting with sterile peritonitis in a patient on peritoneal dialysis to prompt clinicians to consider GCC in the differential diagnosis of similar symptoms in such patients.

\section{Abbreviations}

5-HIAA: 5-hydroxyindoleacetic acid; CT: Computerized tomography; GCC: Goblet cell carcinoid 


\section{Acknowledgements}

None.

\section{Funding}

This study was not supported by any funding.

\section{Availability of data and materials}

The data supporting the conclusions of this article is included within the article.

\section{Authors' contributions}

CWC wrote the manuscript and contributed to acquisition of data. HHC was the treating physician of the patient and contributed to revising critically important intellectual content of the manuscript. JCS performed pathologic analysis and interpretation. LCH performed interpretation of CT image. CCK and $\mathrm{YCL}$ analyzed the clinical course and contributed to conception of conclusion. All authors read and approved the final manuscript.

\section{Competing interests}

The authors declare that they have no competing interests.

\section{Consent for publication}

Written informed consent was obtained from the patient for publication of this case report and any accompanying images. The authors adhered to CARE guidelines/methodology.

\section{Ethics approval and consent to participate}

\section{Not applicable.}

\section{Author details}

'Division of Nephrology, Department of Internal Medicine, Taipei Medical University Hospital, Taipei, Taiwan. ${ }^{2}$ Department of Pathology, School of Medicine, College of Medicine, Taipei Medical University, Taipei, Taiwan. ${ }^{3}$ Department of Medical Imaging, Taipei Medical University Hospital, Taipei, Taiwan. ${ }^{4}$ Translational Imaging Research Center, College of Medicine, Taipei Medical University, Taipei, Taiwan. ${ }^{5}$ Division of Nephrology, Department of Internal Medicine, School of Medicine, College of Medicine, Taipei Medical University, Taipei, Taiwan.

Received: 21 March 2016 Accepted: 8 February 2017

Published online: 13 February 2017

\section{References}

1. Pham TH, Wolff B, Abraham SC, Drelichman E. Surgical and chemotherapy treatment outcomes of goblet cell carcinoid: a tertiary cancer center experience. Ann Surg Oncol. 2006:13(3):370-6.

2. Tang LH, Shia J, Soslow RA, Dhall D, Wong WD, O'Reilly E, Qin J, Paty P, Weiser MR, Guillem J, et al. Pathologic classification and clinical behavior of the spectrum of goblet cell carcinoid tumors of the appendix. Am J Surg Pathol. 2008:32(10):1429-43.

3. Pickhardt PJ, Levy AD, Rohrmann Jr CA, Kende Al. Primary neoplasms of the appendix: radiologic spectrum of disease with pathologic correlation. Radiographics. 2003;23(3):645-62.

4. Akoh JA. Peritoneal dialysis associated infections: an update on diagnosis and management. World J Nephrol. 2012;1(4):106-22.

5. Jaar BG, Plantinga LC, Crews DC, Fink NE, Hebah N, Coresh J, Kliger AS, Powe NR. Timing, causes, predictors and prognosis of switching from peritoneal dialysis to hemodialysis: a prospective study. BMC Nephrol. 2009;10:3.

6. de Freitas DG, Gokal R. Sterile peritonitis in the peritoneal dialysis patient. Perit Dial Int. 2005;25(2):146-51.

7. Martin J, Sansone G, Cirugeda A, Sanchez-Tomero JA, Munoz C, Selgas R. Severe peritoneal mononucleosis associated with icodextrin use in continuous ambulatory peritoneal dialysis. Adv Perit Dial. 2003;19:191-4

8. Vlahakos D, Rudders R, Simon G, Canzanello VJ. Lymphoma-mimicking peritonitis in a patient on continuous ambulatory peritoneal dialysis (CAPD). Perit Dial Int. 1990;10(2):165-7.

9. Bagnis C, Gabella P, Bruno M, Cosseddu D, Marangella M, Yacha GM, Linari F. Cloudy dialysate due to adenocarcinoma cells in a CAPD patient. Perit Dial Int. 1993;13(4):322-3.

10. Roy P, Chetty R. Goblet cell carcinoid tumors of the appendix: an overview. World J Gastrointest Oncol. 2010;2(6):251-8.
11. Gupta A, Patel T, Dargar P, Shah M. Metastatic appendiceal goblet cell carcinoid masquerading as mucinous adenocarcinoma in effusion cytology: a diagnostic pitfall. J Cytol. 2013;30(2):136-8.

12. Subbuswamy SG, Gibbs NM, Ross CF, Morson BC. Goblet cell carcinoid of the appendix. Cancer. 1974;34(2):338-44.

13. Alsaad KO, Serra S, Chetty R. Combined goblet cell carcinoid and mucinous cystadenoma of the vermiform appendix. World J Gastroenterol. 2009; 15(27):3431-3.

14. Anderson NH, Somerville JE, Johnston CF, Hayes DM, Buchanan KD, Sloan JM. Appendiceal goblet cell carcinoids: a clinicopathological and immunohistochemical study. Histopathology. 1991;18(1):61-5.

15. Goede AC, Caplin ME, Winslet MC. Carcinoid tumour of the appendix. Br J Surg. 2003;90(11):1317-22.

\section{Submit your next manuscript to BioMed Central and we will help you at every step:}

- We accept pre-submission inquiries

- Our selector tool helps you to find the most relevant journal

- We provide round the clock customer support

- Convenient online submission

- Thorough peer review

- Inclusion in PubMed and all major indexing services

- Maximum visibility for your research

Submit your manuscript at www.biomedcentral.com/submit
Biomed Central 